\title{
Using the Coefficient of Variation to Improve the Sparsity of Seismic Data
}

\author{
Hasan Al-Marzouqi, Ghassan AlRegib \\ School of Electrical and Computer Engineering \\ Georgia Institute of Technology Atlanta, GA 30332, USA
}

\begin{abstract}
In this work we propose using the coefficient of variation as a cost function to improve seismic data representation in the curvelet domain. Performance improvement is demonstrated in denoising and compressed sensing data recovery. The demonstrated approach can be extended to other seismic applications and alternate transforms.
\end{abstract}

Index Terms-Sparsity, Curvelet, Compressed Sensing

\section{INTRODUCTION}

Directional transforms are gaining popularity in a variety of different applications. In the seismic domain improved performance is reported in areas like denoising, multiple reduction, migration, and fault detection. The Curvelet transform provides one of the most efficient sparse representation of seismic data. The coefficients are generated by taking the inverse fourier transfrom of directional tiles arranged to cover the frequency domain (Fig. 1). Recently, we proposed adapting curvelet tilings to better represent the FFT content in the class of images of interest [1]. Deionising performance, measured in MSE or PSNR, was used as a cost function. NelderMead derivative free method is used to find the optimal tiling locations. In this work we show that improved distribution of curvelet tiles can be obtained by maximizing the coefficient of variation $\left(C_{v}\right)$, where $C_{v}=\frac{\sigma}{\mu}$, of curvelet coefficients magnitude.

\section{SPARSITY MEASURES}

In addition to the coefficient of variation, we tested using shannon's entropy and the Gini index as measures guiding the adaptation process. Shannon's entropy was used by Coifman et. al. [2] to find the best wavelet basis in wavelet packets. The Gini index is a measure of economic statistical dispersion that was recommended for use as a measure of data sparsity in [3]. Note that since curvelet tiling adaptations alter the length of the coefficients vector, minimizing $l^{p}$ norms will not generate satisfactory results.

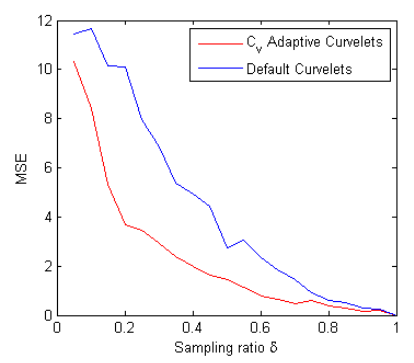

Fig. 2. Seismic data recovery from incomplete measurements

\section{RESUlTS}

A Gaussian noise curvelet denoising algorithm was used to compare the different sparsity measures. Results are summarized in table 1 . Next, the $C_{v}$ optimizer was used to improve seismic data recovery from missing traces. Mathematically, the problem can be formulated as follows [4]:

$\hat{\mathbf{f}}=C^{-1}(\hat{\mathbf{x}}) \quad$ with $\quad \hat{\mathbf{x}}=\arg _{\mathbf{x}} \min \|\mathbf{x}\|_{1} \quad$ subject to $S C^{-1}(\mathbf{x})=\mathbf{b}$

Where $S$ is the sampling matrix that randomly samples $\delta=$ $\frac{n}{N}$ seismic traces. $C^{-1}(\mathbf{x})$ is the inverse curvelet transform of $\mathbf{x}$. The SPGL1 solver was used for data recovery. Fig. 2 summarizes the results obtained.

\section{TABLE I*}

AVERAGE SEISMIC DENOISING PERFORMANCE COMPARISON BETWEEN DEFAULT AND ADAPTIVE TILING CURVELETS. FIVE SEISMIC DATA SETS OF SIZE $550 \times 100$ WERE USED IN THIS EXPERIEMNT.

\begin{tabular}{|c|c|c|c|c|}
\hline & Default & \multicolumn{3}{|c|}{ Adaptive Curvelets } \\
\cline { 3 - 5 } & Curvelets & Entropy & Gini index & $C_{v}$ \\
\hline Avg. MSE $\sigma=1$ & 0.8230 & 0.4930 & 0.4934 & $\mathbf{0 . 4 7 1 0}$ \\
\hline Avg. MSE $\sigma=1.5$ & 1.3600 & 0.9196 & 0.9084 & $\mathbf{0 . 8 2 8 8}$ \\
\hline
\end{tabular}

\section{REFERENCES}

[1] H. Al-Marzouqi and G. AlRegib. "Searching for the optimal curvelet tiling." Acoustics, Speech and Signal Processing (ICASSP), 2013 IEEE International Conference on. IEEE, 2013.

[2] Coifman, Ronald R., and M. Victor Wickerhauser. "Entropy-based algorithms for best basis selection.” Information Theory, IEEE Transactions on 38.2 (1992): 713-718.

[3] Hurley, Niall, and Scott Rickard. "Comparing measures of sparsity." Information Theory, IEEE Transactions on 55.10 (2009): 4723-4741.

[4] Herrmann, Felix J., and Gilles Hennenfent. "Nonparametric seismic data recovery with curvelet frames." Geophysical Journal International 173.1 (2008): 233-248.

Fig. 1. Default curvelet tiling 